\title{
LA REALIDAD RURAL: SITUACIÓN EN UNA REGIÓN. LA ORGANIZACIÓN DE EQUIPAMIENTOS.
}

\section{JESÚS MORAGÓN NAVA}

Trabajador Social. Centro de Salud de Atención Primaria. INSALUD (Albacete).

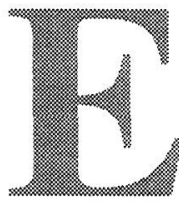

1 presente artículo no pretende ser un estudio exhaustivo de las características definitorias de lo que se entiende por Mundo Rural.

Este documento pretende aportar una visión general sobre algunos aspectos del medio rural, para concretar después esas características en la situación de una región donde lo «rural» tiene un peso importante.

Por último, nos centraremos en cómo la práctica profesional se ve condicionada por las características del medio en el que se desarrolla, haciendo especial referencia a los Centros de Servicios Sociales, como equipamiento básico de atención a la población.

\section{ALGUNOS NÚMEROS SOBRE EL MUNDO RURAL EN ESPAÑA. UNA APROXIMACIÓN.}

La población total de derecho en nuestro país es de 38.872.268 habitantes; 3.079 .079 viven en municipios de menos de 2.000 habitantes y 6.581 .871 en municipios de entre 2.000 y 10.000 habitantes.

Dadas estas cifras, el número de personas que viven en núcleos rurales (considerando como tales los de menos de 10.000 habitantes) es de 9.660 .958 , lo que representa un $24,85 \%$ del total nacional. Por tanto, más de un $75 \%$ de la población vive en núcleos considerados urbanos.

Resulta evidente que el número de personas que vive en zonas rurales es minoritario respecto a la población urbana, si bien se trata de una minoría importante que necesariamente ha de tenerse en cuenta.

Aunque estos son los datos en un momento determinado, censo de 1991, habría que resaltar ciertas tendencias. Se produce una reducción de la población rural, siendo ésta de casi 400.000 habitantes menos que en el año 1986 (según censo de ese año). Así mismo, en el medio 
rural hay más hombres que mujeres, ya que, si bien las cifras globales son muy similares (debido a la mayor esperanza de vida de la mujer), en edades jóvenes las diferencias se acentúan, lo que confirma una mayor tendencia de la mujer rural a salir de su territorio en esas edades.

Otro hecho que resulta determinante, aunque no es exclusivo del medio rural, es el envejecimiento de la población, provocado por un descenso sostenido de la natalidad, así como por el aumento de la esperanza de vida!.

Hasta aquí algunas cifras que nos acercan a la dimensión del fenómeno. Sin embargo, hablar del medio rural es hablar de una realidad compleja, compuesta de diferentes sectores y grupos de población, que, analizados uno a uno, nos proporcionan una visión más detallada de ese mundo. En cualquier caso, ese análisis, hecho de forma individual, es únicamente a efectos de estudio. Esa diversidad hay que entenderla de forma global y en continua interconexión, que es como se da en la realidad.

\section{ACTIVIDAD PRODUCTIVA.}

Al hablar de lo «rural» existe una tendencia a identificarlo con lo «agrario». Esto es así porque históricamente la agricultura ha sido la base de las comunidades rurales. Sin embargo, la situación ha cambiado profundamente y hoy no se puede identificar lo «rural» con lo «agrario» ${ }^{2}$.

El panorama actual nos muestra una agricultura mucho más eficaz, en cuanto al rendimiento, que la que existía hace unos decenios. La actividad agraria se ha modernizado, se ha dado una progresiva mecanización y estamos en un tiempo en el que las altas tecnologías están marcando el ritmo de producción. La modernización de la agricultura no es un fenómeno exclusivo de este momento. Se inició alrededor de los años 60 hasta desembocar en la situación actual. Esa mecanización tiene como consecuencia un incremento importante de la efectividad de las tierras, pero ésta no es la única, y así vemos como cada vez absorbe menos mano de obra, por lo que se produce un desplazamiento de población, hasta entonces ocupada en el sector primario, hacia otros sectores que empiezan a tener una mayor importancia.

Datos obtenidos en «Fondo Documental del Mundo Rural». Tema 1. La Población Rural. Pág. 2. AGANZO TORIBIO, Andrés y Otros. Cáritas Española. Madrid.

? VACA, José María. «Análisis de la realidad rural». Ponencia: La Acción Social de Cáritas en el Mundo Rural. Cáritas Española. 
«La situación de paro es cada vez más acusada y tiende a agravarse. Apuntamos algunas causas:

- El 58\% de los propietarios de las explotaciones tienen 55 años o más.

- Cese de actividad y jubilación anticipada, propuestas desde las nuevas políticas de la CE.

- Cierre de las pequeñas explotaciones familiares, que no pueden ser competitivas a nivel europeo e internacional.

- No existe relevo generacional. A los jóvenes les resulta difícil «permanecer» ante la dureza e incertidumbre por la que atraviesa el sector agrario y las dificultades económicas en la creación de empleos alternativos.

- La preparación y formación personal y profesional es escasa o nula, si se quiere hacer frente a las exigencias que plantea el Mercado Único, la Política Agraria Común (PAC) y los acuerdos del GATT (Acuerdos Generales sobre Aranceles y Comercio), exigencias y acuerdos comerciales entre países. $\gg^{3}$

El análisis de las cifras de población activa en el sector agrario, en relación con lo que representan en el total nacional, vienen a confirmar lo expuesto hasta ahora.

\subsection{Evolución de la población activa en el sector agrario ${ }^{4}$}

Población activa del sector agrario

\begin{tabular}{|llll|}
\hline Año 1974 & $22 \%$ & de la población activa española \\
Año 1980 & $17,8 \%$ & de la población activa española \\
Año 1988 & $14,3 \%$ & de la población activa española \\
Año 1991 & $10,2 \%$ & de la población activa española \\
\hline
\end{tabular}

Dadas las cifras, se aprecia la constante disminución de la población activa en el sector. Además, las previsiones son que la reducción continúe. Ante este descenso ocupacional de la agricultura, en los pueblos se han ido generando una serie de alternativas laborales, ya que si no, estarían condenados a desaparecer. Actualmente, la diversidad laboral es infinitamente mayor que la de hace medio siglo, por lo que ya no es posible identificar lo «rural» con lo «agrario».

3 MARCOS, Purificación. «Manifiesto por un Mundo Rural vivo». Cáritas Española. 1993. Pág. 8.

4 AGANZO TORIBIO, Andrés y Otros. «Fondo documental del Mundo Rural». Tema 8. Los pequeños agricultores. Cáritas Española. Pág. 3.

VACA, José María. «Análisis de la realidad rural». Ponencia: La Acción Social de Cáritas en el Mundo Rural. Cáritas Española. 
Debido a las profundas transformaciones habidas en la producción agrícola, la productividad de las tierras es mucho mayor, sin embargo, la importancia de la riqueza que se genera ha disminuido comparativamente respecto a otros sectores. Los datos nos muestran que:

- En 1972 la agricultura representa el 11,2\% del PNB del país.

- En 1989 representa solamente el 7,5\% del PNB.

Esto se debe a que, aunque la productividad ha aumentado, el crecimiento y la evolución de otros sectores ha sido mucho mayor ${ }^{5}$, por lo que el sector ha perdido peso específico dentro del conjunto de la economía del país, si nos atenemos a las cifras de P.N.B.

\section{III.LA EMIGRACIÓN}

Un hecho, que ya hemos mencionado, estrechamente relacionado con la evolución de la actividad productiva es el de la emigración. Este es un fenómeno paralelo a los procesos de transformación agraria, que aún hoy se sigue produciendo. No se trata de un movimiento que afecte por igual a todos los grupos de edad y sexo, sino que se caracteriza por afectar fundamentalmente a población joven y femenina.

«En el grupo de 14 a 24 años hay 863.686 mujeres y el número de hombres es de 924.776 , lo cual nos indica una mayor tendencia de la mujer rural a salir de su territorio en edades jóvenes» ${ }^{6}$.

Este proceso migratorio, además de la lógica disminución del número de habitantes, trae consigo otra consecuencia, que ahora pasaremos a comentar: el envejecimiento de la población rural.

\section{IV.UNA POBLACIÓN ENVEJECIDA}

El envejecimiento de la población es un hecho que está presente en los países desarrollados, entre ellos España, que ha pasado en los últimos años a la categoría de país envejecido, con más de un $10 \%$ de población considerada anciana. Se trata de un reto para estos países, que han de dar respuesta a las necesidades que surgen en estos grupos de edad.

5 VACA José María. «Análisis de la realidad rural». Ponencia: La Acción Social de Cáritas en el Mundo Rural. Cáritas Española.

" AGANZO TORIBIO, Andrés y Otros. «Fondo documental del Mundo Rural». Tema 1. La Población Rural. Cáritas Española. Pág. 2. 
Algunos datos de lo que supone el fenómeno del envejecimiento en cuanto al presente y el futuro más cercano son: ${ }^{7}$

- En el grupo de edad de 65 y más años, según la proyección realizada por el INSERSO en 1988, para el período 1986 a 2010, observamos un fuerte incremento de este grupo de edad hasta el año 2000 , tendencia que se suaviza desde ese momento hasta el año 2010.

- Los mayores de 80 años aumentan proporcionalmente más que el resto de la población mayor. El ritmo de crecimiento de una población no se incrementa, ésta tiende a envejecer, y esto ocurre también con la población de más edad.

- Mayor presencia de la mujer en estas edades, siendo aún mayor a edades más avanzadas, por lo que se puede hablar de una «ancianidad feminizada».

- En lo que llevamos de siglo se ha duplicado la esperanza de vida de la población al nacer. Actualmente se sitúa alrededor de 80 años para las mujeres y algo menos para los varones.

- En todas las Comunidades Autónomas se ha producido un incremento de la esperanza de vida, sin embargo, los índices de envejecimiento son bastante dispares entre unas y otras. Actualmente, las poblaciones más jóvenes se encuentran en Canarias, País Vasco y Madrid, y las más envejecidas en Aragón, las dos Castillas, Galicia y Extremadura.

- Esta disparidad, que en estos momentos es bastante elevada, tenderá a equilibrarse. Las Comunidades que más van a ver crecer su población anciana van a ser las de Madrid, País Vasco y Canarias, entre otras, mientras que las que presentan una población más envejecida aumentarán su número en menor proporción. La explicación de este fenómeno se debe a que las Comunidades que presentan altos índices de envejecimiento perdieron población en edades jóvenes y adultas a causa de las migraciones, presentando «vacíos demográficos» en determinados grupos de edad, por lo que el incremento de la población anciana será reducido.

Hasta ahora hemos hablado del envejecimiento de la población española en general, pero este fenómeno se agudiza al contemplar los datos de las zonas rurales. «Mientras que los mayores de 65 años de la población total eran 4.689.410, según el padrón de 1986, representando un $12,2 \%$ de la población, los mayores, que según esta misma fuente vivían en municipios de menos de 10.000 habitantes, eran 1.423.942, representando un $14,2 \%$ de la población rural.

7 MUÑOZ, Cristina Catalina. «Los ancianos en el Mundo Rural». Seminario de trabajo, El Escorial, 1991. Cáritas Española. Pág. 3-10. 
De los mayores rurales, 965.468 vivían en municipios de 2.000 a 10.000 habitantes y, el resto, 620.339 en municipios de menos de 2.000 habitantes; en estos municipios la población mayor representa el 19,6\%. Existen núcleos en los que la población de más de 65 años supone entre el 50 y el $100 \% » .{ }^{8}$ Los datos nos aportan otra de las características que definen a los pueblos de nuestra geografía, se trata de núcleos donde el número de personas mayores es comparativamente mucho mayor que en las zonas urbanas.

Esto, que en principio solamente son datos estadísticos, nos muestra que va a ser en estos pequeños núcleos donde surgirán el mayor número de necesidades relacionadas con la ancianidad, por lo que esas Comunidades han de crear los mecanismos necesarios para dar cobertura a las situaciones que lo precisen. Los requerimientos y necesidades de los mayores rurales van a coincidir con los que estos plantean en la sociedad con carácter general, aunque al mismo tiempo, van a presentar características propias del medio en el que habitan:"

- Los procesos de envejecimiento originan, en ocasiones, pérdida de autonomía personal, por lo que la persona pasará a depender de otros.

Al mismo tiempo, suelen convivir únicamente con su cónyuge, o solos ( 3 de cada 4), situación que agravará el proceso de deterioro mencionado.

- En los núcleos rurales, además de las necesidades comunes a todos los mayores, surgen otras relacionadas con la disponibilidad de servicios y recursos, menor que en núcleos no rurales, de tal forma que la solución de determinadas gestiones supone un desplazamiento, $\mathrm{o}$ ante determinadas situaciones problemáticas el anciano ha de trasladarse, con el desarraigo que esto origina. «Como hecho positivo, el anciano rural sigue viviendo en su entorno habitual, en contacto con sus vecinos $y$, en ocasiones, en contacto con actividades de su anterior vida laboral, lo cual facilitará en algunos casos el paso del mundo laboral al mundo jubilado».

\section{OTROS GRUPOS DE POBLACIÓN.}

El análisis podría extenderse a los distintos grupos de población que componen el medio rural. Sin llegar a profundizar, algunas de las

* AGANZO TORIBIO, Andrés y Otros. «Fondo documental del Mundo Rural». Tema 6. Los ancianos y jubilados. Cáritas Española. Pág. 2.

9 AGANZO TORIBIO, Andrés y Otros. «Fondo documental del Mundo Rural». Tema 6. Los ancianos y jubilados. Cáritas Española. Pág. 3-4. 
características que se dan en estos grupos y que complementan la visión sobre la realidad rural son:

- «La ausencia de oferta laboral en el sector industrial ha estabilizado en términos generales la población rural en los últimos años, provocando un aumento del paro.

- Desde la infancia se capta una visión negativa del mundo rural, que provoca una permanente actitud de huida o de obligada resignación.

- Población agraria joven dependiente de los padres hasta edades avanzadas, sin responsabilidad directa en la gestión familiar.

- La composición de la mano de obra agrícola difiere de la de los sectores secundario y terciario, siendo minoritaria la población asalariada frente a la de los trabajadores independientes y familiares». ${ }^{10}$

- En el caso de la mujer, su participación en las actividades agrícolas es, excepto en determinadas Comunidades Autónomas, muy inferior a la del varón. Su actividad productiva se contempla como algo secundario y complementario, estando infravalorada respecto a la actividad del hombre. Por otro lado, en la mayoría de las ocasiones, además de las tareas de explotación, tienen que realizar las faenas domésticas como amas de casa. Este es el grupo de mujeres más numeroso, el que desempeña diversos roles dentro de la actividad familiar. ${ }^{11}$

\section{UNA REGIÓN DONDE LO «RURAL» ES IMPORTANTE.}

Hasta ahora hemos hecho un repaso por algunas de las características que están presentes en el ámbito rural de todo el país. Son circunstancias que se dan en mayor o menor medida en las distintas Comunidades Autónomas, ya que si bien en todas hay núcleos de población de gran importancia, ${ }^{12}$ no es menos cierto que también existe un número más o menos elevado de núcleos pequeños que, en conjunto, daría lugar al panorama descrito con anterioridad.

Por tanto, lo hasta ahora enunciado no es privativo de determinadas Comunidades, sino que es algo propio de lo que se entiende por «MUNDO RURAL», presente por toda nuestra geografía, incluso en aquellas Comunidades con mayores núcleos de población.

10 AGANZO TORIBIO, Andrés y Otros. «Fondo documental del Mundo Rural». Tema 1. La Población Española. Cáritas Española. Pág. 3.

1 AGANZO TORIBIO, Andrés y Otros. «Fondo documental del Mundo Rural». Tema 6. Las mujeres rurales. Cáritas Española. Pág. 3.

12 No hay que olvidar que más del $75 \%$ de la población del país vive en núcleos de más de 10.000 habitantes, siendo la población que vive en núcleos rurales algo menos de un $25 \%$. 
Vamos ahora a analizar en qué medida lo expuesto para definir las zonas rurales tiene su aplicación en una determinada región donde todo esto tiene un peso específico importante, como es Castilla-La Mancha. ${ }^{13}$

Castilla-La Mancha está situada en la sub-meseta meridional de la Península. En extensión es la tercera Comunidad Autónoma del país. Se trata de una región interior con un clima típicamente continental.

En lo referente a la población, ésta representa el 4,4\% del total nacional, mientras que el territorio supone el $15,7 \%$ de la superficie. Se trata de una región con una baja densidad de población, 21 habitantes por kilómetro cuadrado, frente a los más de 74 como media del territorio español. ${ }^{14}$

En cuanto a la distribución, alrededor de 1.000 .000 de personas viven en núcleos de 10.000 habitantes o menos, mientras que son unas 648.000 las que viven en zonas que se consideran urbanas (censo de 1981). A diferencia del resto del país, la población que vive en núcleos considerados rurales (menos de 10.000 habitantes), representa más de un $60 \%$ del total, mientras que para el conjunto español estos núcleos suponen un $24,85 \%$ de la población. Estas cifras muestran la importancia del Mundo Rural dentro de la región.

En lo referente a la evolución que ha experimentado la población, desde 1950 se ha producido un descenso importante y continuado. «Podemos constatar que no sólo se produce una pérdida de población en términos relativos -respecto al conjunto nacional- sino que se ha operado un descenso real de la población, al pasar la población regional de 2.059.700 habitantes en 1960 a 1.648 .600 habitantes el 1-31981 , lo que se traduce en una variación negativa del $20 \%$, mientras que España obtuvo una variación positiva del $34,4 \%$. (...) El origen de esta negativa evolución de la población regional tiene su raíz en los flujos migratorios que ha venido soportando la región y que la han caracterizado como tradicional zona de expulsión». ${ }^{15}$

Actualmente, la tendencia es a la estabilización del movimiento migratorio, al igual que en el resto del territorio español, ya que la crisis económica hace que se produzca una reducción de la demanda

13. Se trata de un ejemplo, donde se pretende mostrar en qué medida lo expuesto en la primera parte del artículo coincide o no para esta determinada región. El análisis podría realizarse sobre otras Comunidades, apareciendo situaciones distintas a las aquí expuestas en determinados apartados, ya que la evolución puede resultar diferente, variando de una zona a otra.

14 CARRIÓN, José y Otros. «Realidades a lo claro, Castilla-La Mancha». Editorial Popular. Pág. 26-36.

15 «Programa de desarrollo regional de Castilla-La Mancha; 1986-1988». Servicio de Publicaciones, Junta de Comunidades de Castilla-La Mancha. Pág. 27-28. 
de mano de obra en las zonas industriales, por lo que hay una mayor tendencia a permanecer en los lugares de origen.

Algo estrechamente relacionado con los movimientos migratorios, y que también caracteriza a esta región, es el envejecimiento de la población.

En general, puede afirmarse que la población castellano-manchega es una población más envejecida que la media nacional ${ }^{16}$. Mientras que en España 14 personas de cada 100 tienen más de 60 años, en Castilla-La Mancha son 19 de cada 100 las personas que están en ese grupo de edad. La constante salida de población de determinados grupos de edad ha provocado, además de una importante pérdida en el número de habitantes, un envejecimiento de la población de la región. Al realizar un análisis de las pirámides de población, se observa un estrechamiento de los grupos de edad más jóvenes, al tiempo que un ensanchamiento en los escalones más elevados ${ }^{17}$ (en las provincias de Cuenca y Guadalajara, la población de más de 60 años representa un $23,4 \%$ y un $22,1 \%$ respectivamente, muy por encima de la media en la región).

Si realizamos un análisis de la población activa en Castilla-La Mancha y su distribución ${ }^{18}$, los datos nos muestran que la mayor parte de la población trabaja en el sector servicios -al igual que en el resto del país-. Después se observa como en agricultura trabaja un $28 \%$ de la población, frente a un $18 \%$ en industria. ${ }^{19}$

Con estos datos, sobre todo al compararlos con los del resto del país, se observa que se trata de una región con una actividad productiva fundamentalmente agraria. Por esto, en la región se han dado los procesos de reconversión y de cambio a los que aludíamos, y que tan importantes modificaciones han ocasionado en el sector. Entre ellos, cabe destacar ${ }^{210}$ :

- Fuerte trasvase de mano de obra del campo a la ciudad ante la aparición de grandes grupos industriales, con las consecuencias que esto origina (envejecimiento poblacional, etc.).

16 CARRIÓN, José y Otros. «Realidades a lo claro, Castilla-La Mancha». Editorial Popular. Pág. 38.

17 «Programa de desarrollo regional de Castilla-La Mancha, 1986-1988». Servicio de Publicaciones, Junta de Comunidades de Castilla-La Mancha. Pág. 29-33.

is CARRIÓN, José y Otros. «Realidades a lo claro, Castilla-La Mancha». Editorial Popular. Pág. 41 .

19 Estos porcentajes se invierten para el conjunto español: la población activa ocupada en el sector industrial es de un $28 \%$, frente a un $18 \%$ en agricultura.

20 «Programa de desarrollo regional de Castilla-La Mancha, 1986-1988». Servicio de Publicaciones, Junta de Comunidades de Castilla-La Mancha». Pág. 33. 
- El sistema de producción agrícola entra en ese proceso de modernización. Se impone la mecanización e importantes transformaciones en los factores de producción (se intenta responder a los nuevos hábitos alimenticios, etc.).

- Se produce un aumento de la superficie de las explotaciones, reduciéndose al tiempo el número de las mismas.

Hasta aquí un repaso por algunas de las situaciones que conforman una parte de la realidad de esta región.

\section{VII.SERVICIOS SOCIALES: SERVICIOS INTEGRADOS EN UNA REALIDAD.}

En el plano de los Servicios Sociales, un hecho que cabe destacar es la extensión/universalización que se ha dado en los últimos años. En la actualidad las prestaciones básicas son un derecho reconocido en todas las Comunidades Autónomas, fruto de la cooperación, a través del Plan Concertado, entre la Administración Central y la Autonómica. Esta mayor presencia de los Servicios Sociales es algo que se ha producido tanto a nivel urbano como en las zonas rurales.

Lo visto hasta ahora son algunas de las situaciones que, si no de manera específica, sí que cobran especial importancia en el medio rural, y que conforman una realidad no exenta de problemas y necesidades. Los Servicios Sociales tratan de dar respuesta a estas necesidades. Lo que resulta evidente es que esa respuesta ha de ser distinta en el medio rural y en el urbano. La concepción de los servicios ha de adecuarse a las características del lugar donde se van a desarrollar, de tal forma que las soluciones que se ofrezcan sean realistas y respondan de manera eficaz a las situaciones de carencia.

Actuaciones que pueden ser acertadas en un determinado espacio, dando lugar a la solución del problema, pueden resultar totalmente ineficaces en otro con una realidad distinta, por lo que han de diseñarse acorde con las peculiaridades del medio, teniendo en cuenta, además, que lo que se entiende por «Mundo Rural» presenta una gran disparidad de situaciones, no tratándose de una realidad homogénea que se extienda a lo largo de nuestra geografía.

«La tipología y manifestación de necesidades sociales en el medio rural es muy variada y requerirá tratamiento acorde con sus características, en igualdad de condiciones respecto al medio urbano, lo que implicará incrementar los esfuerzos en la dotación de equipamientos y que estos tengan un diseño y planificación adecuada a las zonas rurales. 
Es imprescindible que el Sistema de Servicios Sociales articule una completa Red de Equipamientos para dar respuesta a las necesidades de Servicios Sociales.» ${ }^{2 !}$

\section{EQUIPAMIENTOS EN EL MEDIO RURAL.}

La Red de Equipamientos supone el soporte necesario para desarrollar las actividades encaminadas a dar cobertura a las necesidades que se presentan en ese determinado espacio.

Pilar Navío Masegosa y otros definen los equipamientos como «un conjunto estructurado e integrado de recursos físicos, humanos, técni$\cos$, financieros y materiales que permiten llevar a cabo una serie de atenciones y medidas para posibilitar que se hagan efectivas una $o$ varias de las prestaciones del Sistema Público de Servicios Sociales».22

Se trata de una definición con una concepción global de Equipamiento, que abarca todo lo necesario para el desarrollo de las Prestaciones Básicas.

Estos han de ser de diferentes tipos, distinguiendo dos niveles: ${ }^{23}$

- Un primer nivel de equipamientos comunitarios, son los Centros de Servicios Sociales y en ellos estarán ubicadas las unidades de Trabajo Social y los equipos interdisciplinares.

- El segundo nivel hace referencia a los equipamientos específicos. Por tales se entiende los de carácter residencial (viviendas tuteladas, albergues,...), los encaminados a la inserción social (centros ocupacionales, etc.), así como los de relación social (hogares y clubes).

\section{LOS CENTROS DE SERVICIOS SOCIALES.}

Los Centros de Servicios Sociales están encuadrados dentro de ese primer nivel fundamental de equipamientos que hemos definido como Comunitarios, suponiendo la primera referencia para la atención de las demandas planteadas por la población. Entre las funciones a desarrollar, destaca la gestión de las prestaciones básicas, además de la

21 Varios Autores. «Los Centros de Servicios Sociales en el Medio Rural». Dossier Mundo Rural y perspectivas de futuro. Cáritas Española. Pág. 148.

22 VV.AA. «Los Centros de Servicios Sociales en el Medio Rural» Dossier Mundo Rural y perspectivas de futuro. Cáritas Española. Pág 139.

2.3 VV.AA. «Los Centros de Servicios Sociales en el Medio Rurai». Dossier Mundo Rural y perspectivas de futuro. Cáritas Española. Pág. 139. 
función planificadora y de coordinación, sin las cuales no podrían desarrollarse las demás. ${ }^{24}$

Vamos ahora a hacer referencia a los recursos con los que deben contar los Centros, estableciendo las peculiaridades producto del medio en el que están ubicados.

Los recursos mínimos para el correcto funcionamiento de los Centros se estima deben ser los siguientes: ${ }^{25}$

- Instalaciones físicas.

- Otros recursos materiales.

- Plantillas.

- Recursos financieros.

- Técnicas de intervención.

\subsection{Instalaciones físicas.}

No cabe duda que contar con un lugar adecuado para el desempeño de la actividad resulta totalmente necesario.

En el Medio Rural, el profesional, la mayor parte de las veces, no va a desarrollar su trabajo en un único núcleo de población, sino que su actividad va a ser desempeñada en varios núcleos que conforman una determinada zona de actuación. Por esta razón, los profesionales no dispondrán de un único edificio en toda la zona de trabajo, sino que en cada uno de los núcleos tendrán a su disposición las instalaciones necesarias para el desempeño de su actividad.

Los edificios distribuidos por los diferentes municipios de la zona, además de las instalaciones dirigidas a Servicios Sociales, albergarán, en la mayoría de los casos, otros servicios de otras áreas, es decir, se tratará de edificios compartidos.

Este es un hecho propio del Medio Rural, a diferencia del Medio Urbano, donde para el desempeño del trabajo el equipo suele contar con un edificio destinado a tal fin.

Aunque las instalaciones han de ser varias y distribuidas por los distintos municipios, en uno de ellos estará ubicado el edificio de referencia. Este quedará delimitado como la sede administrativa, así como el lugar de confluencia de todos los miembros para la realización de las actividades comunes de programación, coordinación, etc. perspectivas de futuro. Cáritas Española. Pág. 141.

25 VV.AA. «Los Centros de Servicios Sociales en el Medio Rural». Dossier Mundo Rural y perspectivas de futuro. Cáritas Española. Pág. 142-145. 


\subsection{Otros recursos materiales.}

Por las especiales características de movilidad de los profesionales, se deberá pensar en dotar a los centros de:

a) Vehículos. Se entiende que debe formar parte de la dotación del centro. Si esto no fuera posible, se establecerá la compensación económica adecuada por la utilización del vehículo particular.

b) Sistemas de información. Los profesionales trabajan en diferentes lugares, por lo que estos sistemas han de ser ágiles, adaptándose a esta característica del trabajo. Ante esta situación, algunas Comunidades Autónomas están dotando de ordenadores portátiles a sus unidades de Trabajo Social, ya que resulta fundamental tener un fácil acceso a la información en el desempeño de la actividad.

c) Mantenimiento de instalaciones. Ha de tenerse en cuenta que por este tipo de equipamiento, el mantenimiento es sensiblemente más costoso que en Centros urbanos. Esto ha de reflejarse en la elaboración del presupuesto del Centro.

\subsection{Plantillas.}

El número de componentes de las plantillas en los centros estará determinado por la población a atender y el volumen de trabajo que se genere.

Los componentes de las plantillas serán los necesarios para el desempeño de las siguientes funciones:

- Atención directa y gestión de programas.

- La Administración. Se requiere apoyo administrativo a nivel central y en los distintos lugares de trabajo.

- El staf. Dentro del equipo técnico, el Centro contará con un psicólogo y la posibilidad de una consulta jurídica.

- La dirección. La burocracia que se genera en el Centro, es menor que la de otros con mayor número de profesionales, por lo que la persona que realice estas tareas podrá estar implicada en la gestión de programas, etc.

La atención directa, que se presta a través de las unidades de Trabajo Social, presenta en el medio rural algunas situaciones características.

En cuanto al número de habitantes de referencia de una U.T.S., la media en las zonas rurales sería aproximadamente de 5.000, y, si la zona es muy dispersa, menos aún, mientras que ese número estaría muy por encima en las zonas urbanas. El porqué de estas cifras, que supone un mayor número de profesionales para atender una determinada población, viene determinado por dos situaciones: 
1. Los profesionales han de realizar sus funciones en diferentes núcleos de población, con las dificultades de comunicación que esto origina.

2. La demanda que se recibe en el Medio Rural no es en muchas ocasiones específica de Servicios Sociales. Se trata de demandas correspondientes a otras áreas que no tienen centro ubicado en la zona. El Trabajador Social trata de dar respuesta a esos requerimientos en su función de facilitar el acceso a los recursos.

En el medio urbano, existe una mayor facilidad de acceso a los recursos por parte de la población, por lo que el profesional puede canalizar una determinada demanda no específica de Servicios Sociales, sin que esto suponga un desplazamiento para el usuario.

\subsection{Recursos financieros.}

Los Centros de Servicios Sociales en el Medio Rural han de contar con un presupuesto estable que permita garantizar una adecuada atención a la población.

A la hora de presupuestar se ha de tener en cuenta, por las situaciones vistas hasta ahora, que el coste, en términos proporcionales, es sensiblemente superior al de las zonas urbanas.

\subsection{Técnicas de intervención.}

Se han considerado las técnicas como otro de los recursos con los que ha de contar un Centro para el desempeño de su actividad.

Las situaciones a tratar tienen unas determinadas características, por lo que los profesionales ha de desarrollar técnicas de intervención adecuadas para la puesta en práctica en esa zona. En ese sentido, la formación de profesionales ha de adecuarse a las características del medio. «Debemos hacer un esfuerzo por definir esas técnicas en el medio rural, y no mimetizar las del medio urbano que casi siempre terminan en fracaso.»

Se ha hecho especial referencia a los Centros de Servicios Sociales, ya que estos resultan fundamentales, constituyendo la puerta de acceso de los ciudadanos al Sistema Público de prestaciones. Sin embargo, no han de ser los únicos recursos, debiéndose crear todos aquellos equipamientos específicos, dentro del segundo nivel, necesarios para dar cobertura a las necesidades de la zona, acorde con sus peculiaridades. ${ }^{26}$

26. VV.AA. «Los Centros de Servicios Sociales en el Medio Rural». Dossier Mundo Rural y perspectivas de futuro. Cáritas Española. Pág. 140. 


\section{CONCLUSIONES.}

Después de lo visto hasta ahora, no cabe duda que en el Medio Rural se han producido una serie de transformaciones importantes que han cambiado la fisonomía de una realidad, que en estos momentos presenta algunas situaciones preocupantes.

Aunque la población rural continúa descendiendo respecto al total nacional, lo cierto es que constituye una realidad importante, que no puede ser olvidada, y sobre la que se ha de trabajar para potenciar sus capacidades, como se está haciendo en algunos lugares.

Por todo esto, los Servicios Sociales no pueden permanecer ajenos al lugar donde se encuentran y, en ese sentido, constituyen otro de los elementos que han de contribuir al desarrollo de la zona, intentando dar cobertura a las distintas necesidades, en una realidad no exenta de ellas.

Lógicamente, la organización de los mismos ha de presentar unas características propias, de cara a responder de forma eficaz a esa problemática, y aunque el coste por persona es ligeramente superior, resultan totalmente necesarios para que no se acentúen las diferencias entre dos realidades que presentan situaciones tan distintas.

\section{BIBLIOGRAFÍA}

AGANZO TORIBIO, A. y otros: «Fondo documental del Mundo Rural». Cáritas Española. Madrid.

CARRIÓN, J. y otros: «Realidades a lo claro, Castilla-La Mancha». Editorial Popular. Madrid.1987. $2^{\mathrm{a}}$ edición.

MARCOS, P.: «Manifiesto por un Mundo Rural vivo». Cáritas Española. Madrid. 1993.

MUÑOZ, C.C.: «Los ancianos en el Mundo Rural». Seminario de Trabajo, El Escorial, 1991. Cáritas Española.

«Programa de desarrollo regional de Castilla-La Mancha, 1986-1988». Servicio de Publicaciones, Junta de Comunidades de Castilla-La Mancha. Toledo. 1986.

VACA, J.M.: «Análisis de la realidad rural». Ponencia: La Acción Social de Cáritas en el Mundo Rural. Cáritas Española. 1989.

Varios autores: «Los Centros de Servicios Sociales en el Mundo Rural». Dossier Mundo Rural y perspectivas de futuro. Cáritas Española. Madrid. 1990. 